\title{
IONOGEL-BASED LIGHT-ACTUATED VALVES FOR CONTROLLING LIQUID FLOW IN MICROFLUIDIC MANIFOLDS
}

\section{Fernando Benito-Lopez, ${ }^{1}$ Robert Byrne, ${ }^{1}$ Ana Maria Răduță, ${ }^{1}$ Nihal Engin Vrana, ${ }^{2}$}

\section{Garrett McGuinness $^{2}$ and Dermot Diamond ${ }^{1 *}$}

${ }^{1}$ CLARITY: Centre for Sensor Web Technologies, National Centre for Sensor Research, School of Chemical Sciences, Dublin City University, Dublin 9, Ireland.

${ }^{2}$ School of Mechanical and Manufacturing Engineering Material Processing and Research Centre, Dublin City University, Dublin 9, Ireland.

E-mail: fernando.lopez@dcu.ie, robert.byrne@dcu.ie, radutana@gmail.com,

evrana8@gmail.com, garrett.mcguinness@dcu.ie, dermot.diamond@dcu.ie

* Author to whom correspondence should be addressed; Tel.: + 353-1-700 7937; Fax:

$+353-1-7007995$

\section{ILLUSTRATED CONTENTS LIST}

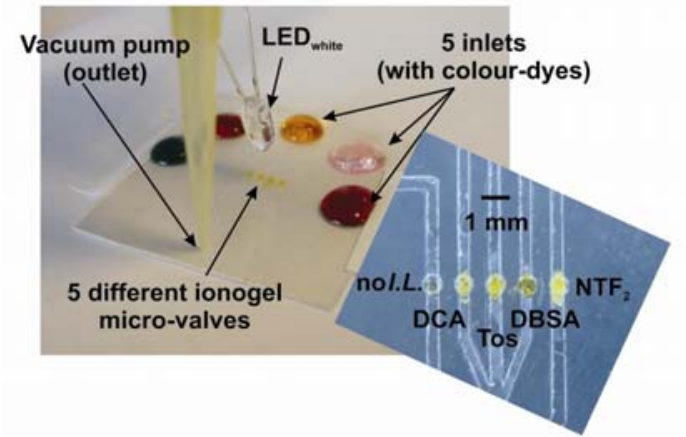

Novel multifunctional materials based on ionogels have been used as light-actuated valve structures in micro-fluidic platforms. Through variation of the composition of the ionogels, the micro-valves can be tuned to open at different times under similar illumination conditions. 


\begin{abstract}
We present the fabrication, characterisation and performance of four novel ionic liquid polymer gels (ionogels) as photo-actuated valves incorporated into microfluidic manifolds. The ionogels incorporate benzospiropyran units and phosphonium based ionic liquids. Each ionogel is photo-polymerised in-situ in the channels of a poly(methyl methacrylate) micro-fluidic device, generating a manifold incorporating four different micro-valves. The valves are actuated by simply applying localised white light irradiation, meaning that no physical contact between the actuation impulse (light) and the valve structure is required. Through variation of the composition of the ionogels, each of the micro-valves can be tuned to open at different times under similar illumination conditions. Therefore, flows through the manifold can be independently controlled by a single light source. At present, the contraction process to open the channel is relatively rapid (seconds) while the recovery (expansion) process to re-close the channel is relatively slow (minutes), meaning that the valve, in its current form, is better suited for single-actuation events.
\end{abstract}

Keywords: micro-fluidic device; ionic liquids; spirobenzopyran; photoresponsive polymer, micro-valve, photoactuation. 


\section{Introduction}

Micro-fluidic devices are extensively used in many different fields like biomedical [1], pharmaceutical research [2], chemical reaction manifolds [3, 4] and for chemical/bio-analysis [5]. In these devices, valves are essential for the control and movement of flows in the microchannels, while simultaneously minimising dead volume. Applications of micro-valves include flow regulation, on/off switching and sealing of liquids, gases or vacuums. Micro-valves have been developed in the form of active or passive structures employing mechanical, non-mechanical and external systems [6].

In the case of passive valves, which do not have any moving component and are easy to fabricate, hydrogel based materials have received special attention. These valves change volume in response to external stimuli to stop/open the flow [7-11]. Although hydrogels can directly convert chemical energy into mechanical work,[12] which eliminates the requirement of external power source, the fabrication of hydrogels in microfluidics with precise control of position and composition is challenging [13]. In particular, valves made using photo-responsive gels are of great interest as functional materials within micro-fluidic systems since actuation can be controlled by simple light irradiation, without physical contact, offering improvements in versatility during manifold fabrication, and control of actuation [9, 14-16].

A new concept in materials science is the incorporation of ionic liquids (ILs) within polymer gels, to produce materials known as ionogels. Ionic liquids are attracting much attention because of potential applications in many fields of chemistry 
and industry due to their chemical and thermal stability, low vapor pressure, high ionic conductivity properties, and tuneable hydrophobic and hydrophilic nature [1719]. They have also been labelled as 'designer solvents [20], because it is possible to tailor anions and/or cations for specific functions such as catalysis, solubility and viscosity. Incorporating ILs into polymer gels is also attractive as it may generate materials with the inherent advantages of ILs within a solid or semi-solid gel-type structure.

We recently reported the photo and solvatochromic properties of nitrobenzospiropyran and spirooxazine in ionic liquids containing the anion bis(trifluoromethylsulfonyl)imide $\left[\mathrm{NTf}_{2}\right]^{-}[21,22]$ It was found that the kinetics and thermodynamics of the spirobenzopyran-merocyanine equilibrium was sensitive to the nature of the cation. It was also observed that the imidazolium cation, ethylmethylimidazolium $[\mathrm{emim}]^{+}$can even form a through-space orbital interaction with the merocyanine isomer, rather than a simple electrostatic interaction, thus inhibiting the merocyanine conversion back to the spirobenzopyran isomer. The IL tetradecyl(trihexyl)-phosphonium bis(trifluoromethylsulfonyl)-imide, also showed similar kinetic rates of ring closing, which was not expected. We investigated this further, by changing the anion of phosphonium based salts and monitoring the photochromic behaviour of benzospiropyran. It was found that the spectroscopic data obtained in phosphonium ILs contradicted what was expected for benzospiropyran in molecular solvents, with the evidence suggesting that the probe molecule was experiencing multiple solvation shells.[23]

Within the IL field, phosphonium-based ILs possess some interesting and often advantageous properties. When compared with the popular imidazolium based 
ILs, phosphonium cations lack the acidic proton which leads to carbene formation,[24] and are often less dense than water when compared to nitrogen based ILs. More importantly, it is well known that some phosphonium ILs are more thermally stable than their nitrogen based counterparts. Through the tailoring of chemical and physical properties of ILs, robustness, acid/ base character, viscosity and other critical operational characteristics can be finely adjusted. Therefore, we can tune the characteristics of the ionogels by changing the IL and so more closely control the physical and chemical properties of these novel materials.

In this paper we present the incorporation and characterisation of four autonomous photo-responsive phosphonium based ionogel micro-valves in an hybrid poly(methyl methacrylate) (PMMA) pressure-sensitive adhesive (PSA) micro-fluidic device. The valves are fabricated using a novel material consisting of a polymeric structure incorporating benzospiropyran units and diverse ionic liquids. The microvalves are actuated by simply applying localised white light irradiation. By using different IL components within the gels, it is possible to tune the valve actuation time and so independently control liquid flow within the channels of the micro-fluidic device under common illumination conditions, facilitating sequential actuation events

\section{Experimental Section}

\subsection{Materials}

$N$-isopropylacrylamide (NIPAAm, Wako) was purified by recrystallisation in a mixed solution of hexane and toluene and dried under a vacuum. 1',3',3'-Trimethyl6-hydroxyspiro(2H-1-benzopyran-2,2'-indoline) (Acros Organics, Geel, Belgium), acryloyl chloride (Wako), N,N-methylene-bis(acrylamide) (MBAAm, Wako), 2,2- 
dimethoxy-2-phenyl acetophenone (DMPA, Sigma-Aldrich, St. Louis, USA), and 1buthanol (Wako) were used without further purification. Trihexyltetradecylphosphonium dicyanoamide $\left[\mathrm{P}_{6,6,6,14}\right][\mathrm{dca}]$ (abbreviated: [dca $]^{-}$), trihexyltetradecylphosphonium bis(trifluoromethanesulfonyl)-amide $\quad\left[\mathrm{P}_{6,6,6,14}\right]\left[\mathrm{NTf}_{2}\right] \quad$ (abbreviated: $\left.\left[\mathrm{NTf}_{2}\right]^{-}\right)$, trihexyltetradecyl- phosphonium dodecylbenzenesulfonate $\left[\mathrm{P}_{6,6,6,14}\right][\mathrm{dbsa}]$ (abbreviated: [dbsa $]^{-}$), and triisobutyl(methyl)phosphonium tosylate $\left[\mathrm{P}_{1,4,4,4}\right][$ tos] (abbreviated: [tos] ${ }^{-}$), obtained compliments of Cytec Industries. All the ILs were purified thoroughly by column chromatography,[25] dried under vacuum at $40{ }^{\circ} \mathrm{C}$ for $48 \mathrm{~h}$, and stored under argon at $20{ }^{\circ} \mathrm{C}$. PMMA slides were purchased form Goodfellow, UK and 50 um double-sided Pressure Sensitive Adhesive film (AR8890, Adhesives Research, Ireland). The fabrication of the micro-fluidic devices was carried out using a Laser Ablation system-Excimer/ $\mathrm{CO}_{2}$ laser, Optec Laser Micromachining Systems, Belgium.

White (430-760 nm), LEDs were purchased from Roithner Laser Technik, Austria. These were operated under a stabilised constant current of $25 \mathrm{~mA}$ generated by an in-house made power supply. The UV light source used for photopolymerisation was a BONDwand UV-365 nm obtained from Electrolyte Corporation, USA. UV-Vis spectra were recorded on a UV-Vis-NIR Perkin-Elmer Lambda 900 spectrometer and profilometer experiments were carried out in a Veeco Dektak V200SL Profilometer, USA. Microvalve shrinking motion was followed using

a CCD camera coupled to a Prism and Reflector Imaging Spectroscopy System (PARISS) from Lightform Inc., USA.

\subsection{Preparation of photo-responsive phosphonium based ionogels.}


The ionogels consist of three monomeric units; poly( $N$-isopropylacrylamide), $N, N$-methylene-bis(acrylamide) and acrylated benzospiropyran in the ratio 100:5:1, respectively. Figure 1a shows a PMMA mould with reservoirs of $1 \times 5 \mathrm{~mm}$ and 500 $\mu \mathrm{m}$ depth where the different ionogels were polymerised for actuation study using contact profilometer measurements and Figure $1 \mathrm{~b}$ presents the chemical structure of the ionic liquids used in this study. The acrylated benzospiropyran is synthesised as described elsewhere [26]. A reaction mixture solution placed in the micro-fluidic reservoir was prepared by dissolving the NIPAAm monomer (452 $\mathrm{mg}, 4.0 \mathrm{mmol}$ ), the MBAAm (10.8 mg, $0.07 \mathrm{mmol})$, acrylated spirobenzopyran monomer (14.0 mg, 0.04 mmol), and the photo-initiator dimethoxy-phenylacetophenone DMPA (10.2 mg, 0.04 mmol) into 1-buthanol (1.0 mL). These monomers were photo-polymerised within the different ionic liquid matrixes using a UV irradiation source $(365 \mathrm{~nm})$ placed $8 \mathrm{~cm}$ from the monomers. When the polymerisation was completed, the gels were washed with ethanol and $0.1 \mathrm{mM} \mathrm{HCl}$ aqueous solution for $10 \mathrm{~min}$ to remove any unpolymerised liquid and any excess ionic liquid. Finally the ionogels were immersed for two hours in $0.1 \mathrm{mM} \mathrm{HCl}$ aqueous solution, in which the ionogel exhibits a drastic and rapid swelling effect. 


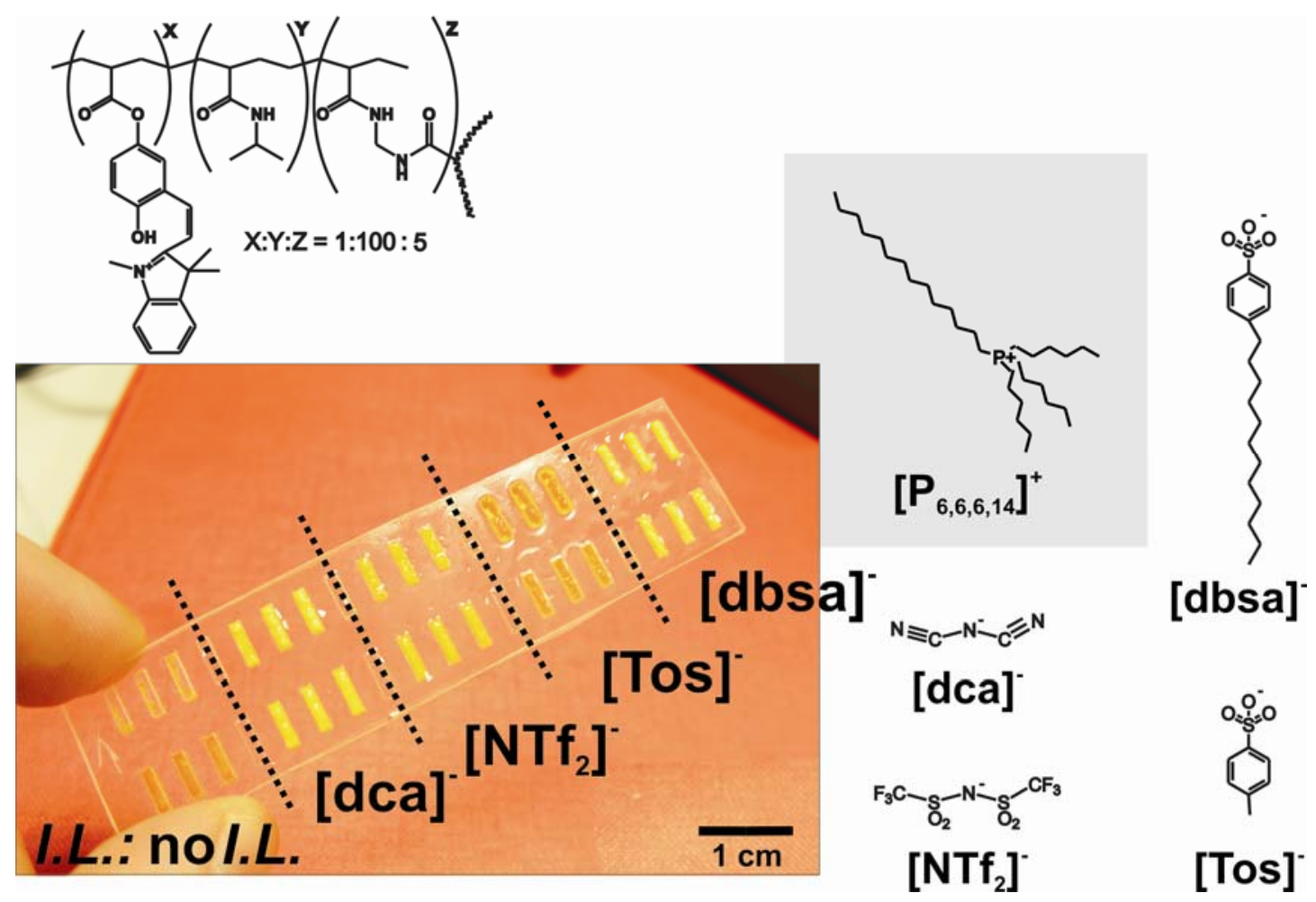

(a)

(b)

Figure 1: a) Chemical structure of the photo-responsive ionogel (top left) and four ionogels polymerised in a $\left(\begin{array}{llll}1 & \times & 5 & \mathrm{~mm}\end{array}\right)$ PMMA mould. b) trihexyltetradecylphosphonium based ionic liquid chemical structures.

\subsection{Micro-fluidic device fabrication}

The micro-fluidic device shown in Figure $2 \mathrm{a}(4 \mathrm{x} 4 \mathrm{~cm})$, consisting of five independent micro-channels with a common outlet, was easily fabricated in poly(methyl methacrylate) and pressure-sensitive adhesive in four layers using a $\mathrm{CO}_{2}$ ablation laser. The ionogel valves were placed in a circular reservoir (250 $\mu \mathrm{m}$ radius), also fabricated using the $\mathrm{CO}_{2}$ laser, within the PMMA $(125 \mu \mathrm{m})$ and the PSA $(50 \mu \mathrm{m})$ thick layers. A second PSA layer incorporating the channel structures (50 $\mu \mathrm{m}$ deep, 
$500 \mu \mathrm{m}$ width) was fabricated using the $\mathrm{CO}_{2}$ laser and glued to the previous PSA layer. Finally the upper PMMA layer, which contains the inlets and outlet, completes and seals the micro-fluidic structure, Figure $2 b$.

The micro-valve actuation reproducibility experiments were carried out using a similar micro-fluidic device $(4 \mathrm{x} 4 \mathrm{~cm})$ but with five parallel channels for independent valve actuation control. The ionogel valve is placed in a square reservoir $(500 \times 500 \mu \mathrm{m})$, while channel dimensions and fabrication procedure were the same as for the previous device.

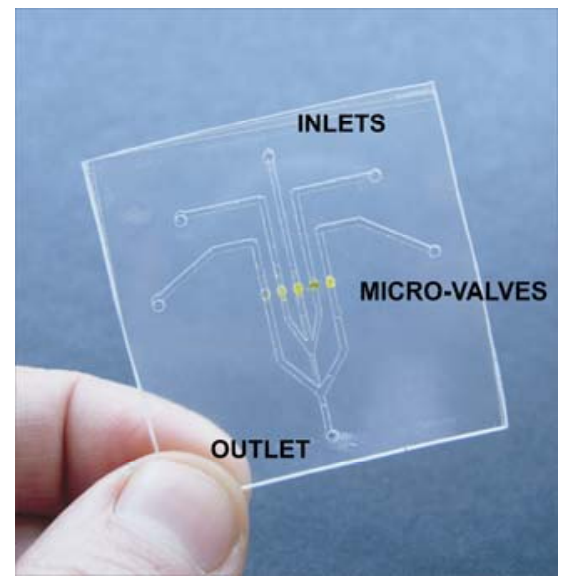

(a)

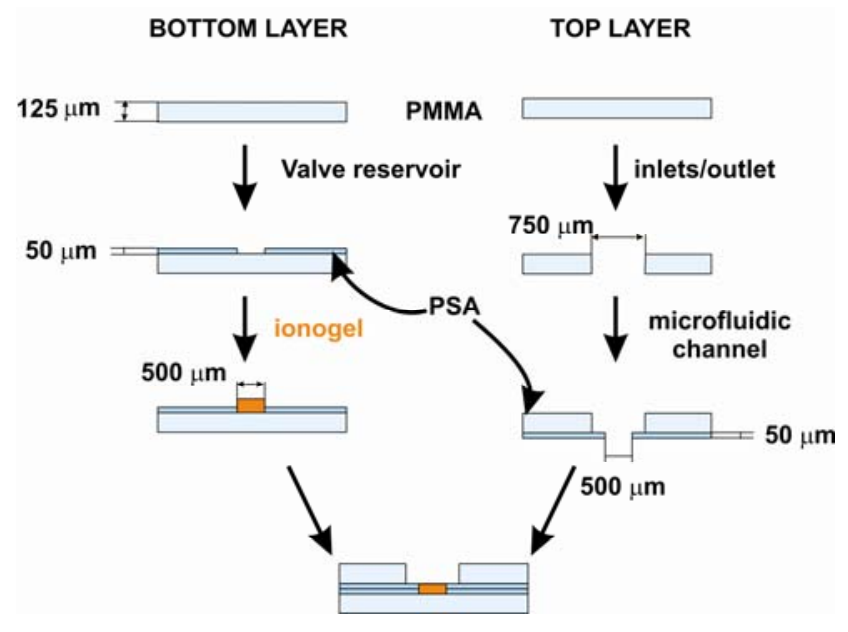

(b)

Figure 2: a) Picture of the micro-fluidic device fabricated in PMMA:PSA polymer by $\mathrm{CO}_{2}$ laser ablation. The first valve on the left contains the photoresponsive polymer gel with no IL [15] while in the other four, the polymer gel has been photopolymerised within $[\mathrm{dca}]^{-},[\mathrm{tos}]^{-},[\mathrm{dbsa}]^{-},\left[\mathrm{NTf}_{2}\right]^{-}$, respectively. b) Schematic representation of the microfluidic device fabrication procedure. 


\subsection{Characterisation of the photo-responsive phosphonium based ionogels.}

The volume phase transition behaviour of the photo-responsive phosphonium based ionogels was investigated using white light irradiation. The physical shrinking by photo-induced dehydration of the ionogel was measured using contact profilometry experiments when white light was applied. To achieve this, a small part of the mould shown in Figurela, containing the ionogel, was placed in the chamber of the Dektak 200-Si, Veeco contact Profilometer which had been modified to incorporate a white light LED source, which was operated using a forward current set at $25 \mathrm{~mA}$, for online ionogel actuation studies. Firstly, the ionogel, immersed in a $10 \mathrm{mM} \mathrm{HCl}$ aqueous solution, was irradiated with white light for a known period of time (irradiation intervals of $3 \mathrm{~s}$ ) and secondly the ionogel was automatically located below the profilometer tip for profiling measurements (ionogel height) as shown in the supplementary information. Optical microscopy observation of the ionogel performance was carried out using a PARISS: "Prism and Reflector Imaging Spectroscopy System” equipped with a CCD camera.

\subsection{Evaluation of ionogel micro-valve performance.}

In order to evaluate the micro-fluidic device performance of the ionogel micro-valves, different coloured dyes solutions, each at $1 \mu \mathrm{M}$ concentration, in water were placed in the inlet reservoirs while in the outlet, a constant vacuum pressure was applied ( ca. 0.69 bar) as a driving force. When visible light was applied using the same LED light source $5 \mathrm{~mm}$ from the valve $(25 \mathrm{~mA})$, the ionogel rapidly decreased in volume and the valve opened, allowing the coloured dye pass through the channel. The time for the micro-valve to open (i.e. for the dye to pass across the valve structure) we define as the valve actuation response time. Images of the system were 
taken every two seconds with a digital camera for monitoring the liquid movement through the micro-channels.

\section{Results and Discussion}

\subsection{Characterisation of photo-responsive phosphonium based ionogels.}

In order to evaluate the feasibility of applying photo-responsive phosphonium based ionogels to micro-valves, rectangular-shaped structures were prepared by photo-polymerisation in a $1 \times 5 \mathrm{~mm}$ PMMA mould. Figure $3 \mathrm{a}$ shows the photoresponsive polymer gels generating with the same amount of monomer, and after immersion of the mould in a $0.1 \mathrm{mM} \mathrm{HCl}$ solution for two hours. [15] By simple visual inspection it was possible to observe significant differences in volume, roughness, shape and colour between the ionogels and the reference polymer gel which did not contain any ionic liquid.

Figure 3 b-c presents two snapshots (time: 0s (b) and time $2 \mathrm{~s}(\mathrm{c}))$ taken from a video, which illustrates the shrinking process when constant white light irradiation is applied to the [dca] ionogel. It was observed that after two seconds of exposure the ionogel size decreases by almost $30 \%$. (See supplementary information video.1) 


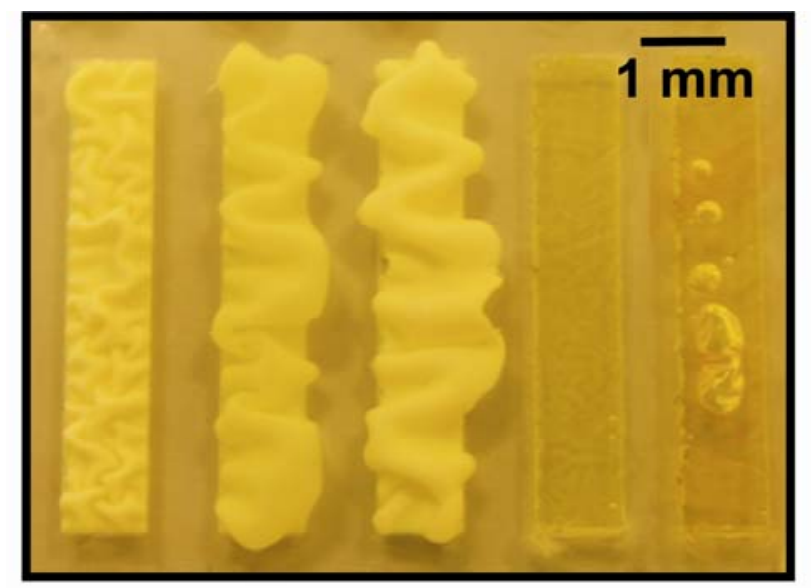

[dbsa] [NTf $]_{2}^{-}$[dca] [Tos] no I.L.

(a)

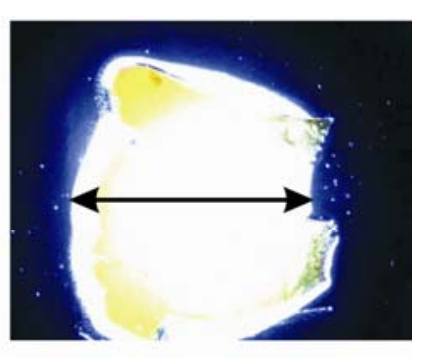

(b)

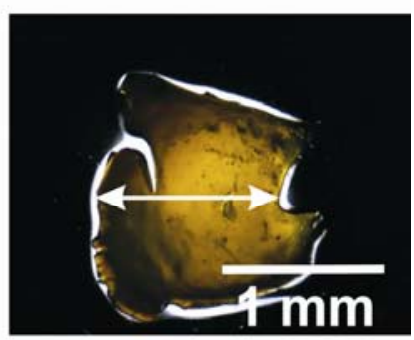

(c)

Figure 3: a) Photo-responsive polymer gels after immersion of the mould in a $1 \mathrm{mM}$ $\mathrm{HCl}$ solution for two hours. Left: [dca] ionogel shrinking process: (b) ionogel before illumination and (c) the same sample after two seconds illumination with a white light LED, size decrease is ca. $30 \%$ by volume.

The ring-closing mechanism of the benzospiropyran moiety in the ionogel was studied for the [dca] ionic liquid in order to gain insight into how this process leads to subsequent expulsion of water and restructuration of the ionogel, i.e. the molecular processes that underlies the observed bulk shrinkage behaviour.[27, 28] Figure 4 shows the UV-Vis analysis of the [dca] $]^{-}$ionogel upon visible light irradiation. At equilibrium, the protonated form of the spirobenzopyran-merocyanine can be identified from the absorbance band at $440 \mathrm{~nm}$. Upon exposure to visible light, this feature decreases in intensity due to the ring closing of the protonated merocyanine (MC- $\mathrm{H}^{+}$, hydrophilic) and formation of the spirobenzopyran (SP, hydrophobic) form. This leads to loss of the acidic proton, thus generating a much more hydrophobic 
environment, which in turn drives the expulsion of water. The process follows firstorder kinetics, with a rate constant estimated as ca. $2.5 \times 10^{-2} \mathrm{~s}^{-1}$ for the [dca] ionogel, (Figure 1 inset).

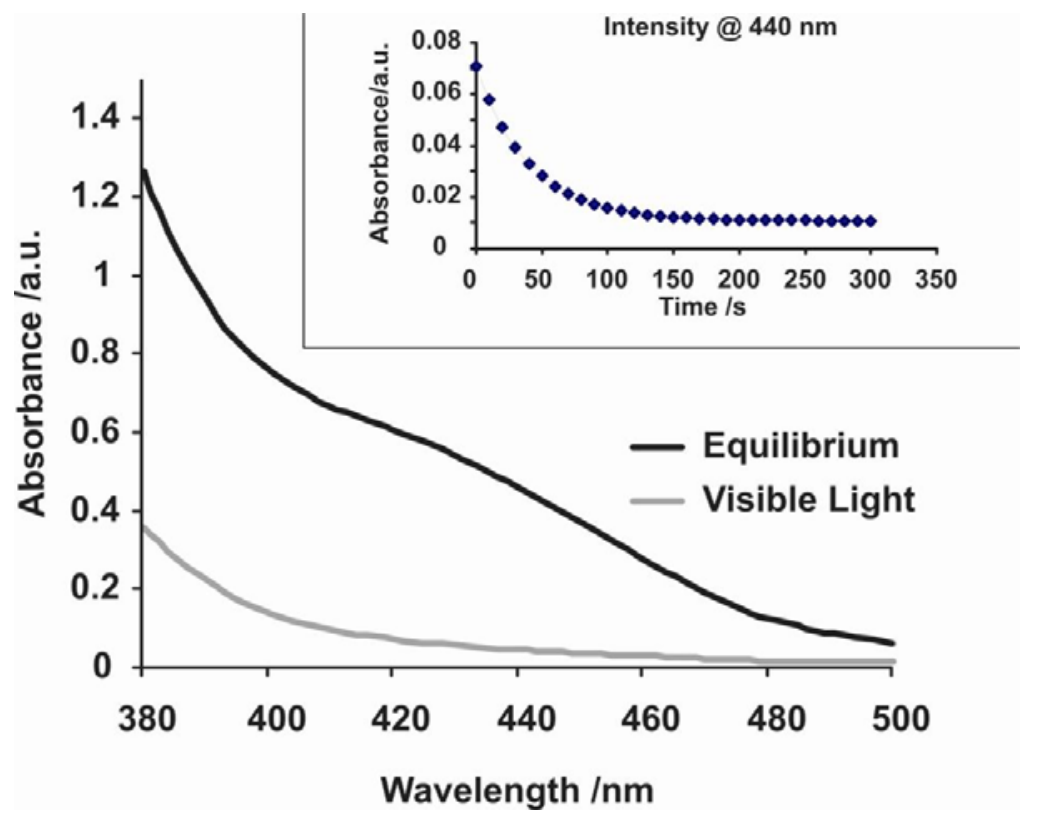

Figure 4: UV-Vis spectrum of the [dca] ionogel at equilibrium and after $60 \mathrm{~s}$ exposure to visible light. (Inset) First order kinetic plot of absorbance decay at 440 nm under visible light irradiation.

Before irradiation, the ionogels have a yellowish colour due to the presence of the protonated merocyanine isomer $\left(\lambda_{\max }=440 \mathrm{~nm} \text { in [dca }\right]^{-}$ionogel). Samples of $c a$. $2 \mathrm{~mm}$ diameter of the various ionogels were irradiated with white light using a LED source placed $5 \mathrm{~mm}$ from the ionogel (see supplementary information for the set-up and ionogel colour behaviour, video.2). The protonated merocyanine isomer, which is responsible for the yellowish colour of the ionogel, exhibits different kinetics for the $\mathrm{MC}-\mathrm{H}^{+} \Rightarrow \mathrm{SP}+\mathrm{H}^{+}$switching process due to the nature of the anion present within the ionogel. We have previously reported that merocyanine/spiropyran (MC/SP) ring- 
closing kinetics in certain ILs can be altered by varying the anion, in some cases by an order of magnitude.[22, 23] For example, the life-time of the MC isomer in phosphonium based ILs containing $[\mathrm{dca}]^{-}$is five times shorter than that of a phophonium IL containing the [tos] $]^{-}$anion. This ring-closing mechanism is the basis of the ionogel deformation, therefore different anion composition should have a dramatic affect on the actual actuation response of the ionogels [28, 29], Figure 5.

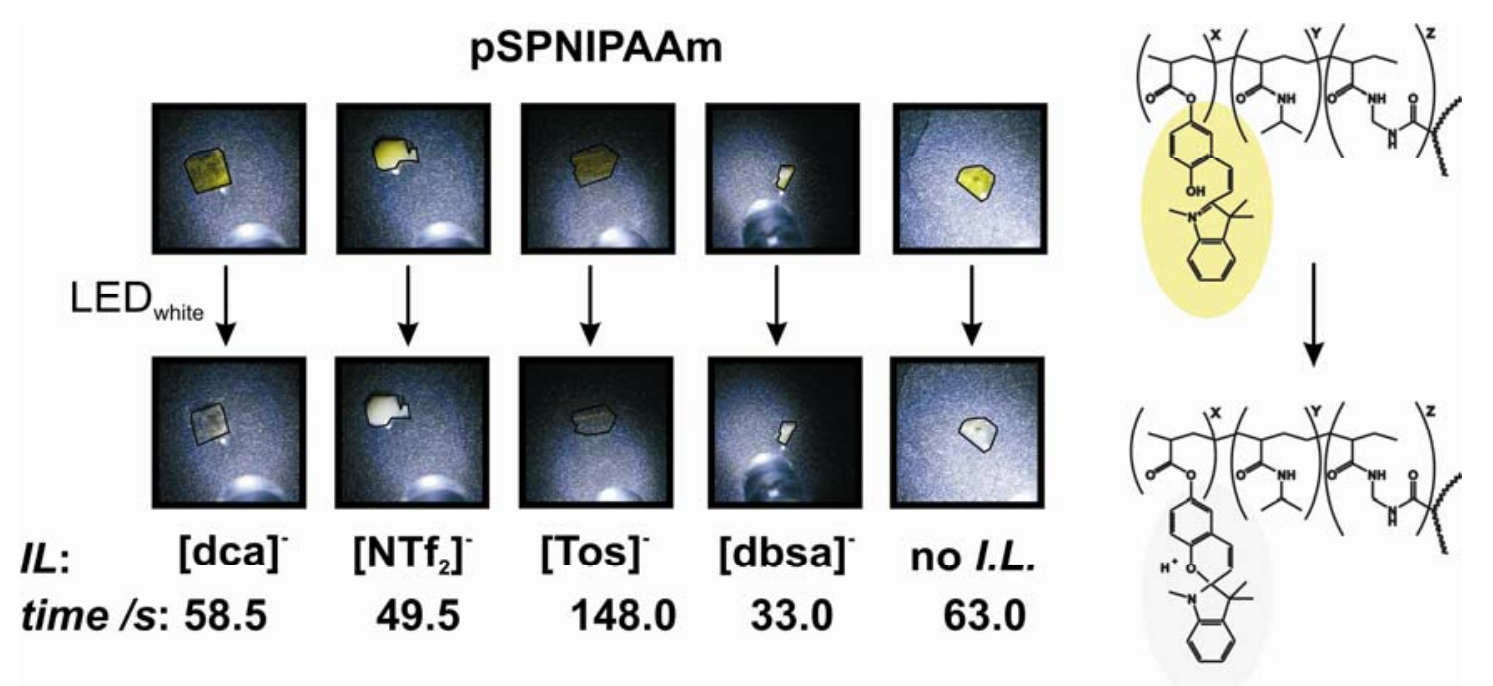

Figure 5: The photographs illustrate the yellow colour evanescence during white light irradiation using the LED of several ionogel pieces before (top) and after (bottom) complete isomerisation from the $\mathrm{MC}-\mathrm{H}^{+}$to the closed-ring SP form. (Time data error: $\pm 2 \mathrm{~s})$.

Since photo-induced dehydration results in a physical shrinkage of the ionogel, as seen in the images in the Figure $3 b-c$, this effect was studied for the various ionogel samples and the IL-free polymer gel using contact profilometry as described in the experimental section 2.4. Different actuation behaviour was observed for each of the ionogels (Figure 6, see supplementary information for reproducibility 
experiments). After 100 seconds of visible light irradiation, the shrinking process was essentially complete. The fastest actuation, with associated decrease in ionogel height with time, and most obvious physical shrinkage happens in the case of the IL-free polymer gel. This gel has a simple polymeric matrix, and therefore, upon formation of the SP moiety, water expulsion and polymer restructuring occurs very rapidly (more than $15 \%$ decrease in height after $100 \mathrm{~s}$ irradiation). In the case of the ionogels, where the matrix structure is much more complex, the ionic liquid plays a crucial role in regulating the water loss and restructuration of the polymer which accompanies formation of the SP moiety. For instance, the [dca] ionogel shows the fastest and most impressive actuation of the ionogels, which correlates well with previous work, which suggests that the $[\mathrm{dca}]^{-}$anion does not interact with the ring open (MC) isomer therefore does not inhibit the light induced ring closure.[23] More importantly, it has been reported previously that swelling and de-swelling of hydrogels is severely affected by the local $\mathrm{pH}$. This is due to the disruption of intermolecular hydrogen bonding of polymer branches by strong acid/bases, and the induction of a partialionisation of the polymer back-bone, which leads to a greater affinity for water molecules and therefore a larger volume. It has been stated in the literature that the anion [dca] $]^{-}$behaves similarly to a Lewis base, and in fact this anion has been used in base-catalysed acetylation reactions. This chemical property is unique to the [dca] ionogel in the ILs studies in this investigation, and this could explain the difference in actuation behaviour observed. For example, if we compare the [dca $]^{-}$ionogel to the $\left[\mathrm{NTf}_{2}\right]^{-}$ionogel, which has similar ring-closing kinetics, the actuation observed was much slower, almost negligible compared to the [dca] ionogel, Figure 6. However, an investigation into the physical properties of the $\left[\mathrm{NTf}_{2}\right]^{-}$ionogel, Table 1 and supplementary information, showed it to be very stiff, and the dehydration and 
restructuration processes are therefore substantially inhibited in the $\left[\mathrm{P}_{6,6,6,14}\right]\left[\mathrm{NTf}_{2}\right] \mathrm{IL}$ matrix. The mechanical properties of the $\left[\mathrm{NTf}_{2}\right]^{-}$ionogel indicate that the anion plays a significant role in determining the overall polymer structure, and it has been reported recently that anions play a major role in the swelling of polymer gels.[30] It is known that $\left(\left[\mathrm{P}_{6,6,6,14}\right]\left[\mathrm{NTf}_{2}\right]\right.$ is very hydrophobic and therefore practically unhydrated to begin with. We previously reported that the $\left[\mathrm{NTf}_{2}\right]^{-}$anion does not interact with the $\mathrm{MC}$ form, and therefore does not hinder the ring-closing $\mathrm{MC} \Rightarrow \mathrm{SP}$ process. [21]

Table 1. Axial Stiffness, Ultimate Tensile Strength (UTS) and Elongation at break values for the ionogels.

\begin{tabular}{cccc}
\hline Ionogel & $\begin{array}{c}\text { Axial Stiffness } \\
/ \mathrm{Nmm}^{-1}\end{array}$ & UTS /MPa & Elongation at Break /\% \\
\hline$[\mathrm{dbsa}]^{-}$ & 0.1713 & 0.12 & 187.19 \\
No $\left.I . L .^{\mathrm{tos}}\right]^{-}$ & 0.0493 & 0.08 & 65.910 \\
[dca $^{-}$ & 0.0187 & 0.02 & 545.48 \\
[NTf $\left._{2}\right]^{-}$ & 0.0149 & 0.02 & 131.53 \\
\hline
\end{tabular}

However, it is known in the literature that the anion composition can control the phase-transition temperature upon which this actuation mechanism depends, and it has been reported that for some ILs containing the $\left[\mathrm{NTf}_{2}\right]^{-}$anion, the phase transition temperature is substantially increased from $\sim 32^{\circ} \mathrm{C}$ to $>100^{\circ} \mathrm{C}$.[31] Therefore the hydrophilic to hydrophobic effect that accompanies the $\mathrm{MC}-\mathrm{H}^{+} \Rightarrow \mathrm{SP}+\mathrm{H}^{+}$process is almost completely swamped by the high residual hydrophobic nature of the $\left[\mathrm{P}_{6,6,6,14}\right]\left[\mathrm{NTf}_{2}\right]$ IL itself. This interpretation is supported by the fact that this ionogel reduces in height by less than $3 \%$ after $100 \mathrm{~s}$ irradiation with white light. On the other hand, the $[\mathrm{dca}]^{-}$based ionogel, which is very flexible and water permeable, produces the greatest effect of all the micro-valves, reducing in height by more than $10 \%$ after irradiation for $100 \mathrm{~s}$ with white light. In comparison, the [tos] ${ }^{-}$and [dbsa $]^{-}$ionogels 
show decreases in height of $8 \%\left([\mathrm{tos}]^{-}\right)$and $6 \%\left([\mathrm{dbsa}]^{-}\right)$. It appears that the more flexible ionogels can undergo faster restructuring and therefore faster actuation, than is observed with stiffer ionogels, Table.1. Phosphonium salts containing the [tos] ${ }^{-}$and [dbsa]" anions have been classed as "inert" hydrophobic solvents [32], and therefore they both should not interact significantly with the $\mathrm{MC}-\mathrm{H}^{+}$isomer, and this should affect the resulting physico-chemical properties of the gel. This was also observed previously in solution experiments.[23] However, it was previously reported by Iwata that aromatic ionic liquids can form $\pi-\pi$ complexes with aromatic solutes.[33] This might explain the difference between the two observed kinetic responses (Figure 6), as the [tos] $]^{-}$anion would be expected to have a stronger interaction with the $\mathrm{MC}-\mathrm{H}^{+}$ isomer than the more sterically hindered $[\mathrm{dbsa}]^{-}$anion.

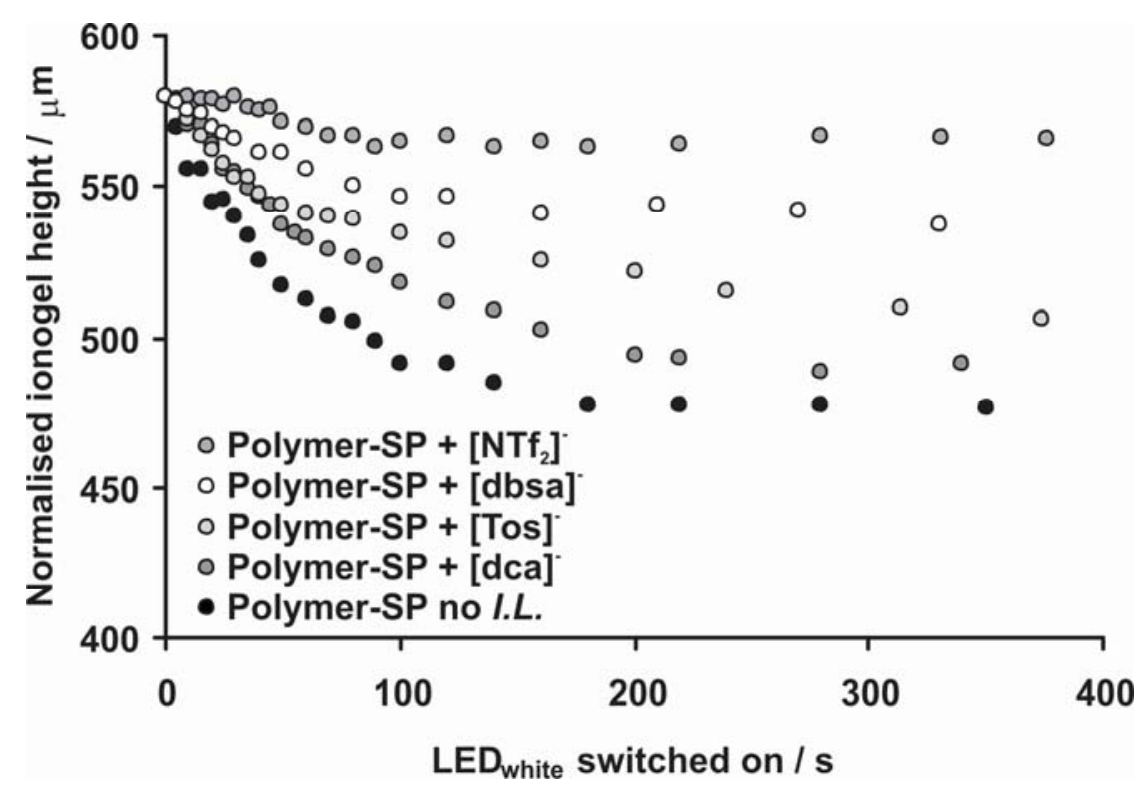

Figure 6: Response kinetics of ionogels upon irradiation with white light (ionogel height error: $\pm 5 \mu \mathrm{m})$. 
The results suggest that, as in the case of photo-responsive polymer hydrogels, $[27,28]$ the photo-induced ionogel shrinkage proceeds through two different steps; at first, the $\mathrm{MC}-\mathrm{H}^{+} \Rightarrow \mathrm{SP}+\mathrm{H}^{+}$photoswitching process takes place under white light irradiation, and this is rapidly followed by water expulsion from the bulk polymer from the resulting much more hydrophobic SP-ionogel. Therefore, these two processes are the responsible of the divergence in actuation behaviour of the different ionogels when irradiated with the same amount of white light.

\subsection{Evaluation of photo-responsive phosphonium based ionogel micro-valves.}

Control of photovalve actuation time by varying the ionogel composition under similar local light irradiation was demonstrated using the micro-fluidic device shown in Figure 2a and the protocol explained in section 2.5 (Figure 7a). Virtually equal amounts of white light were irradiated locally to all ionogel micro-valves and they were found to open at different times as shown in Figure.8b-f. The IL-free polymer gel microvalve opens $2 \mathrm{~s}$ after initial irradiation, followed by the [dca] based ionogel (4 s,), the [tos] based ionogel (18 s), then the [dbsa] based ionogel (44 s), and finally, the $\left[\mathrm{NTf}_{2}\right]^{-}$based ionogel (49 s). The results demonstrate that local light irradiation allows the independent control of multiple micro-valves in a micro-fluidic device, while the synthesis of ionogels with different ionic liquids enables valve actuation at different times, under similar illumination conditions. This approach clearly facilitates non-contact operation and flexible manipulation of liquid movement in complex micro-fluidic devices, as well as parallel control of multiple micro-valves with a single light source. For example, where a microfluidic system is exposed to sunlight, a sequence of actuation events can be generated through the use of valves based on differing ionogel compositions. 
The light intensity necessary to control the ionogel micro-valve is relatively mild. For example, a simple LED with an output of $c a .1 \mathrm{~mW} \mathrm{~cm}^{-2}$ [34] can be used to actuate the valve, compared to previously reported optically controlled micro-valves employing a nanocomposite hydrogel witch used either green light, $1.6 \mathrm{~W} \mathrm{~cm}^{-2}$, or near-IR light, $2.7 \mathrm{~W} \mathrm{~cm}^{-2}$.[35] In fact, the required light intensity is so low that inexpensive off-the-shelf LEDs can be employed rather than expensive laser equipment for controlling valve actuation.

The $\mathrm{MC}-\mathrm{H}^{+} \Rightarrow \mathrm{SP}+\mathrm{H}^{+}$photoswitching process is a reversible phenomenon, as the SP form returns spontaneously to the $\mathrm{MC}^{-} \mathrm{H}^{+}$form, which induces re-swelling of the ionogel. Therefore, in principle it is possible to reuse the micro-valves repeatedly by simply refilling the channels with a $10 \mathrm{mM} \mathrm{HCl}$ water solution, although in its current form, the ionogel requires more than 30 min to swell sufficiently to re-block the channel. However, we found that was possible to successfully reuse the microvalves three times in a microfluidic-device. Further attempts to repeat the open/close valve operation failed due to inability to completely re-seal the channel, although considerable swelling and contraction was still occurring. At present, therefore, the valves are optimum for single use although continued development of the ionogel composition and design of the valve structures should result in improved open/close actuation times. It is important to stress that opening of the valve does not require complete contraction of the valve - a relatively small contraction can open the channel, allowing the dye to pass through, and actuation times for closed to open can be relatively fast (a few seconds).

Micro-valve actuation reproducibility experiments were performed using micro-fluidic manifolds that had five independent channels each with an incorporated 
micro-valve. The results showed that the IL free micro-valves presented reproducible and very fast light actuation response times, but their fabrication was not as straight forward as for the micro-valves incorporating ionic liquids. This resulted in high rejection rates, and only ca. $60 \%$ micro-valves were found to be functional after complete micro-fluidic device fabrication, Figure 8a. The reason for this is that the monomeric solution is not particularly stable within organic solvents, and after a couple of hours, the monomer start to precipitate in solution. This issue is compounded by the fact the organic solvent is volatile. In contrast, in the case of the phosphonium ILs, the monomers are highly soluble within the ILs, and because of their inherit non-volatility; there are no fluctuations in monomer/IL wt\%. Furthermore, it is known that ILs have higher polymer loading capacities than their organic solvent counterparts, and for these reasons, the IL-based gels are generally more easily processable than their IL-free counterparts.[36] 

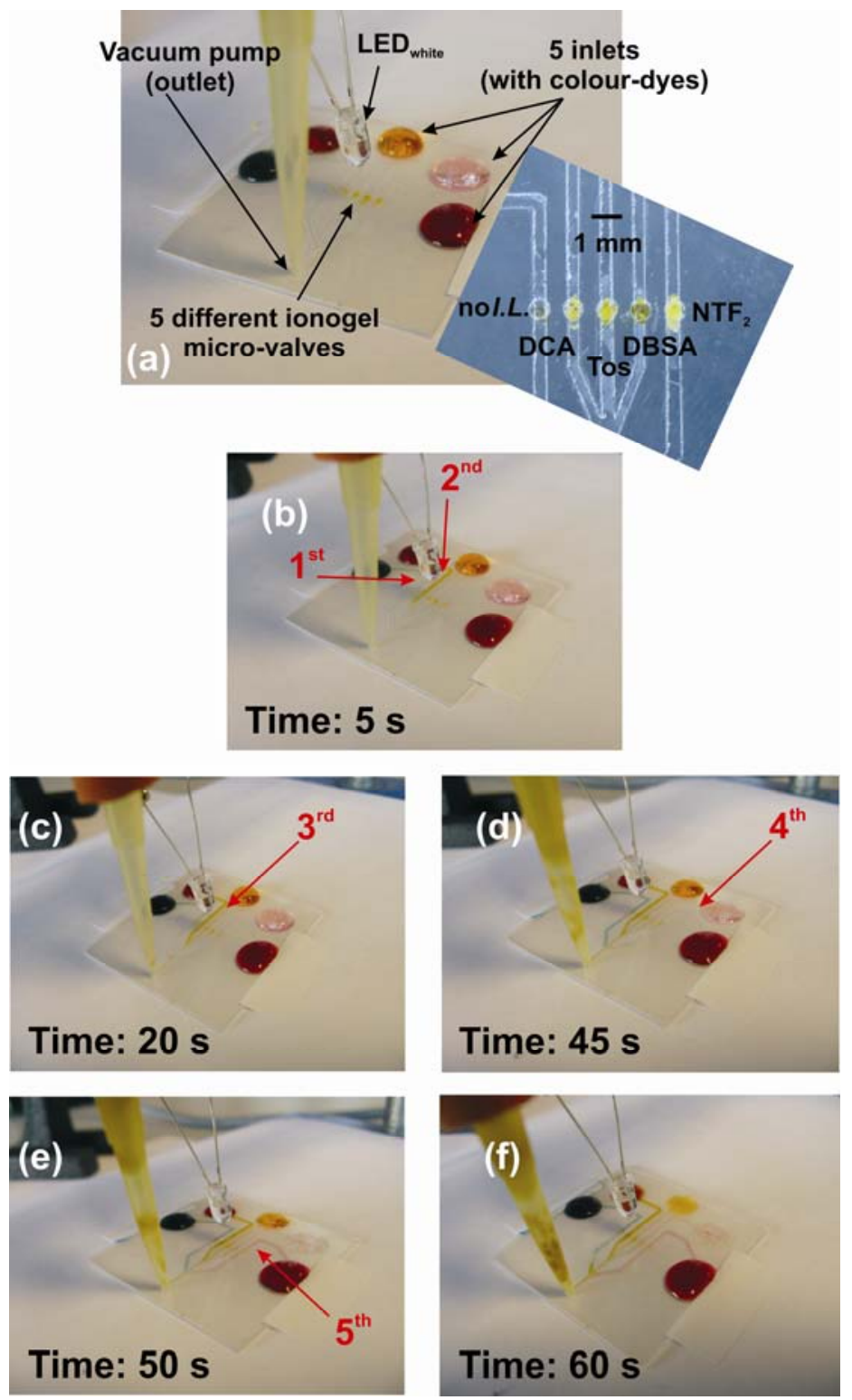

Figure 7: Performance of the ionogel micro-fluidic valves: a) Micro-valves closed; the applied vacuum is unable to pull the dyes through the micro-channels. White light is applied for the time specified in each picture b) 'No I.L.' valve is first to actuate followed by ionogels incorporating [dca $]^{-}(\mathbf{c}),[\mathrm{tos}]^{-}(\mathbf{d}),[\mathrm{dbsa}]^{-}(\mathbf{e}),\left[\mathrm{NTf}_{2}\right]^{-}(\mathbf{f})$, all valves are open. Numbers and arrows indicate when the channel is filled with the dye because of micro-valve actuation.

The ionogel micro-valves also have reproducible photo-actuation response times within a particular type, but their response times vary significantly from anion to 
anion. For instance, the $[\mathrm{dca}]^{-}$based ionogel has a very fast response time of approx. 4 $\mathrm{s}$, while the equivalent $\left[\mathrm{NTf}_{2}\right]^{-}$ionogel responds much more slowly under the same conditions (ca. $50 \mathrm{~s})$.

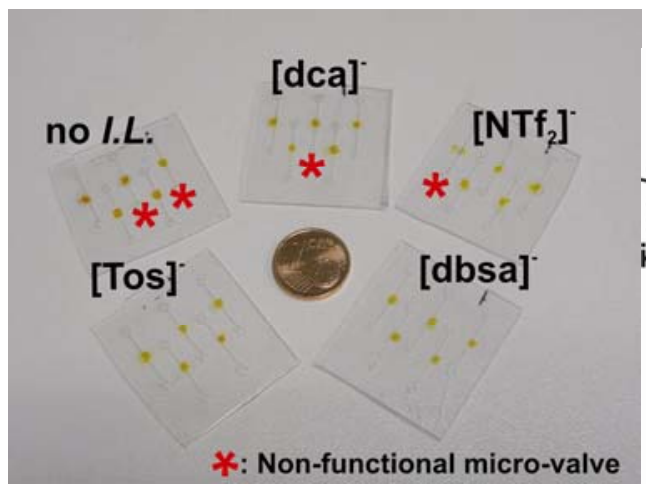

(a)

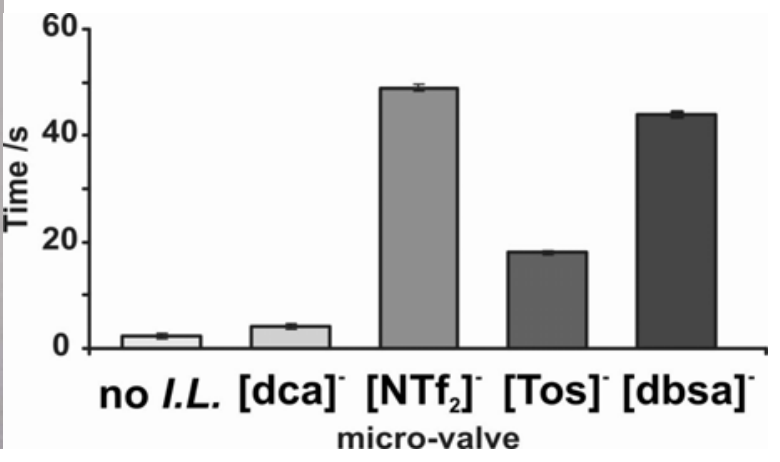

(b)

Figure 8: a) Five micro-fluidic devices with micro-valves. The red start represents micro-valves that were not functional. b) micro-valve actuation response times and their errors (average of at least four functional micro-valves).

By simply varying micro-fluidic valve design, large numbers of micro-valves can be fabricated simultaneously using the process described above and therefore, the method is suitable for large-scale integration of micro-valves into micro-fluidic manifolds. Photo-control of micro-valve actuation facilitates non-contact operation and independent manipulation of liquids in multiple microfluidic channels within an integrated microfluidic manifold. It is clear that such photoresponsive ionogel microvalves have the potential to greatly enhance the ease of fabrication and subsequent operation of multifunctional micro-fluidic devices [37].

\section{Conclusions}


Novel photo-responsive phosphonium based ionogels have been prepared, characterised and compared with an equivalent IL-free hydrogel. It was found that upon irradiation with visible light the ionogels decreased in volume due to $\mathrm{MC}-\mathrm{H}^{+}$ $\Rightarrow \mathrm{SP}+\mathrm{H}^{+}$switching, and associated loss of water from the polymer. These ionogels were then successfully incorporated into a micro-fluidic device as photo-actuated micro-valves.

It was shown that these micro-valve ionogels presented fast actuation responses, (in some cases a few seconds to go to the 'open' valve state), and that the micro-valves based on differing IL anions opened at differing times under similar white light illumination conditions. The results demonstrated that localised light irradiation allows the independent control of multiple micro-valves, while the synthesis of ionogels with different ILs enables valve actuation at different times under common illumination conditions. While it is possible to reuse the valves several times, times to re-achieve valve closure are at present relatively long (ca. 30 minutes) and require contact with an acidic solution. Therefore these valves are at present best suited for applications that involve single use (disposable) devices. However, they do offer very considerable advantages compared to electronically actuated valves in that there is no physical contract required between the actuation signal and the valve structure. Clearly, therefore, they facilitate flexible, low-cost, low power control of liquid movement in complex micro-fluidic devices. Moreover, as rapidly expanding libraries of ionic liquids are becoming generally available, the tailoring of chemical and physical properties of associated ionogels such as physical robustness, acid/ base character, and viscosity can be finely adjusted. Therefore we expect that the actuation characteristics of the ionogels can be further optimised by variation of the ionogel composition. 


\section{Acknowledgements}

The project has been carried out with the support of the Irish Research Council for Science, Engineering and Technology (IRCSET) fellowship number 2089, Science Foundation Ireland under grant 07/CE/I1147 and EU FP6 project MEST-CT-2005-020621.

\section{References}

1. S. Nagrath, L. V. Sequist, S. Maheswaran, D. W. Bell, D. Irimia, L. Ulkus, M. R. Smith, E. L. Kwak, S. Digumarthy, A. Muzikansky, P. Ryan, U. J. Balis, R. G. Tompkins, D. A. Haber and M. Toner, Nature, 2007, 450, 1235-U1210.

2. P. S. Dittrich and A. Manz, Nature, 2006, 5, 210-218.

3. L. S. Bouchard, S. R. Burt, M. S. Anwar, K. V. Kovtunov, I. V. Koptyug and A. Pines, Science, 2008, 319, 442-445.

4. M. Brivio, W. Verboom and D. N. Reinhoudt, Lab. Chip, 2006, 6, 329-344.

5. J. West, M. Becker, S. Tombrink and A. Manz, Anal. Chem., 2008, 80, 44034419.

6. K. W. Oh and C. H. Ahn, J. Micromech. Microeng., 2006, 16, R13-R39.

7. J. Kim, J. Baek, K. Lee, Y. Park, K. Sun, T. Lee and S. Lee, Lab. Chip, 2006, 6, 1091-1094.

8. C. W. Liu, J. Y. Park, Y. G. Xu and S. Lee, J. Micromech. Microeng., 2007, 17, 1985-1991.

9. D. Kim and D. J. Beebe, Sens. Actuators, A, 2007, 136, 426-433.

10. J. Wang, Z. Y. Chen, M. Mauk, K. S. Hong, M. Y. Li, S. Yang and H. H. Bau, Biomed. Microdevices, 2005, 7, 313-322.

11. N. S. Satarkar, W. L. Zhang, R. E. Eitel and J. Z. Hilt, Lab. Chip, 2009, 9, 1773 1779 .

12. J. M. G. Swann and A. J. Ryan, Polym. Int., 2009, 58, 285-289.

13. A. Chunder, K. Etcheverry, G. Londe, H. J. Cho and L. Zhai, Colloids Surf., A, 2009, 333, 187-193.

14. G. F. Chen, F. Svec and D. R. Knapp, Lab. Chip, 2008, 8, 1198-1204. 
15. S. Sugiura, K. Sumaru, K. Ohi, K. Hiroki, T. Takagi and T. Kanamori, Sens. Actuators, A, 2007, 140, 176-184.

16. J. M. Park, Y. K. Cho, B. S. Lee, J. G. Lee and C. Ko, Lab. Chip, 2007, 7, 557564.

17. C. M. Forsyth, D. R. MacFarlane, J. J. Golding, J. Huang, J. Sun and M. Forsyth, Chem. Mater., 2002, 14, 2103-2108.

18. P. Wasserscheid, Recent Developments in Using Ionic Liquids as Solvents and Catalysts for Organic Synthesis. Organic Synthesis Highlights V. 2003; pp 105117.

19. C. Chiappe and D. Pieraccini, J. Phys. Org. Chem., 2005, 18, 275-297.

20. K. Seddon, TCE, 2002, 33-35.

21. R. Byrne, K. J. Fraser, E. Izgorodina, D. R. MacFarlane, M. Forsyth and D. Diamond, Phys. Chem. Chem. Phys., 2008, 10, 5919-5924.

22. O. Coleman, R. Byrne, S. Minkovska and D. Diamond, Phys. Chem. Chem. Phys., 2009, 11, 5608-5614.

23. R. Byrne, S. Coleman, K. J. Fraser, A. Raduta, D. R. MacFarlane and D. Diamond, Phys. Chem. Chem. Phys., 2009, 11, 7286-7291.

24. S. Chowdhury, R. S. Mohan and J. L. Scott, Tetrahedron, 2007, 63, 2363-2389.

25. A. K. Burrell, R. E. Del Sesto, S. N. Baker, T. M. McCleskey and G. A. Baker, Green Chem., 2007, 9, 449-454.

26. A. Szilagyi, K. Sumaru, S. Sugiura, T. Takagi, T. Shinbo, M. Zrinyi and T. Kanamori, Chem. Mater., 2007, 19, 2730-2732.

27. K. Sumaru, M. Kameda, T. Kanamori and T. Shinbo, Macromolecules, 2004, 37, 7854-7856.

28. K. Sumaru, K. Ohi, T. Takagi, T. Kanamori and T. Shinbo, Langmuir, 2006, 22, 4353-4356.

29. K. Sumaru, M. Kameda, T. Kanamori and T. Shinbo, Macromolecules, 2004, 37, 4949-4955.

30. X. B. Hu, J. Huang, W. X. Zhang, M. Li, C. G. Tao and G. T. Li, Adv. Mater., 2008, 20, 4074-4078.

31. T. Ueki and M. Watanabe, Macromolecules, 2008, 41, 3739-3749.

32. J. M. Pringle, M. Forsyth, G. G. Wallace and D. R. MacFarlane, Macromolecules, 2006, 39, 7193-7195. 
33. K. Iwata, H. Okajima, S. Saha and H. O. Hamaguchi, Acc. Chem. Res., 2007, 40, 1174-1181.

34. S. Stitzel, R. Byrne and D. Diamond, J. Mater. Sci., 2006, 41, 5841-5844.

35. S. R. Sershen, G. A. Mensing, M. Ng, N. J. Halas, D. J. Beebe and J. L. West, Adv. Mater., 2005, 17, 1366-1368.

36. S. D. Zhu, Y. X. Wu, Q. M. Chen, Z. N. Yu, C. W. Wang, S. W. Jin, Y. G. Ding and $\mathrm{G}$. Wu, Green Chem., 2006, 8, 325-327.

37. S. Sugiura, A. Szilagyi, K. Sumaru, K. Hattori, T. Takagi, G. Filipcsei, M. Zrinyi and T. Kanamori, Lab. Chip, 2009, 9, 196-198. 